\title{
Villous Adenocarcinoma
}

National Cancer Institute

\section{Source}

National Cancer Institute. Villous Adenocarcinoma. NCI Thesaurus. Code C4142.

An adenocarcinoma characterized by the presence of a villous architectural pattern. It may arise from a villous adenoma. 